\title{
Titanium Nitride - a correlated metal at the threshold of a Mott transition
}

\author{
H. Allmaier, ${ }^{1,}$ \& L. Chioncel,,${ }^{2,3}$ and E. Arrigoni ${ }^{2}$ \\ ${ }^{1}$ Institute of Theoretical and Computational Physics, \\ Graz University of Technology, A-8010 Graz, Austria \\ ${ }^{2}$ Institute of Theoretical Physics, Graz University of Technology, A-8010 Graz, Austria \\ ${ }^{3}$ Faculty of Science, University of Oradea, RO-47800, Romania
}

\begin{abstract}
We investigate electron correlation effects in stoichiometric Titanium Nitride (TiN) using a combination of electronic structure and many-body calculations. In a first step, the Nth-order muffin tin orbital technique is used to obtain parameters for the low-energy Hamiltonian in the $T i$ - $d\left(t_{2 g}\right)$-band manifold. The Coulomb-interaction $U$ and the Hund's rule exchange parameter $J$ are estimated using a constrained Local-Density-Approximation calculation. Finally, the many-body problem is solved within the framework of the Variational Cluster Approach. Comparison of our calculations with different spectroscopy results stresses the importance of electronic correlation in this material. In particular, our results naturally explain a suppression of the TiN density of states at the Fermi level (pseudogap) in terms of the proximity to a Mott metal-insulator transition.
\end{abstract}

\section{INTRODUCTION.}

Transition metal-nitrides have been studied for several decades due to their appealing properties, such as ultra-hardness, high melting point and high Curietemperature. This combination of physical and chemical characteristics makes them particularly suitable for coating applications. These materials exhibit metallic conductivity and some of them even show superconductivity, as for example the $\mathrm{Nb}$ based carbonitride ${ }^{\underline{1}}$ which has a transition temperature of $18 \mathrm{~K}$. Recent low-temperature transport properties of thin TiN superconducting films $\stackrel{2}{2}$ show a disorder-driven transition from a superconductor to an insulating phase in which superconducting correlations persist. These experiments performed on homogeneously disordered TiN films clearly demonstrate the important role of electronic correlations. For these reasons, one could expect that signatures of many-body effects might be also present in bulk, stoichiometric TiN.

Electronic properties of bulk transition metal nitrides have been investigated using X-ray Photoelectron Spectroscopy (XPS) $)^{3.4}$, Ultraviolet Photoemission Spectroscopy (UPS $)^{\frac{5}{}}$, X-ray emission ${ }^{6}$, BremsstrahlungIsochromat Spectroscopy (BIS) $\underline{\underline{7}}$, and Electron-Energy-

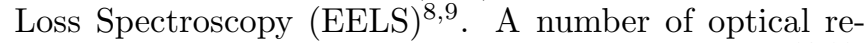
flectivity measurements have also been carried out 10,11 . On the theoretical side, a large number of band structure calculations using density functional theory (DFT) - mostly within the local-density-approximation (LDA) - is present in the literature $\mathrm{3}^{3,12,13,14}$. LDA results describe bonding in terms of (i) a metallic contribution giving a finite density of states at the Fermi level, (ii) an ionic contribution caused by the charge transfer from the metal to the non-metal atom, and (iii) a covalent contribution due to the interaction between the non-metal $p$ and the metal $d$ valence states in addition to the (iv) metal-metal interactions. In the case of TiN, it is believed that bonding is mostly covalent in origin. However, while the LDA results for the occupied part of the density of states (DOS) show a good agreement with spectroscopic data at high binding energies, they fail to describe correctly the energy range in the vicinity of the Fermi energy (see, e. g., Fig. 2) where predominantly Ti-3d states are present $15,16,17$. In particular, a suppression of the XPS spectrum over a range of energy down to $\approx 1 \mathrm{eV}$ below the Fermi energy, could be explained so far only by means of an artificial broadening, whose parameters are optimized to fit the experimental data. In this paper, we present an alternative view and argue that this suppression can be naturally explained in terms of correlation effects, and, specifically, by the proximity to a Mott metal-insulator transition.

Recent developments in the methodology of electronicstructure calculations allow to go beyond the LDA and include electronic correlation effects, which are particularly important for electrons in $d$ or $f$ orbitals. The simplest mean-field extension of LDA is the LDA+U approach $\frac{18}{}$. However, as we show below, even such extension is not sufficient to improve the agreement with experiments in TiN. For this reason, it is necessary to go beyond the mean-field approach and to appropriately deal with dynamical correlation effects. In this paper, we carry out this task by means of a cluster-perturbative method, the Variational Cluster Approach (VCA) ${ }^{19}, 20$, which we combine with LDA in order to obtain the appropriate model parameters.

This paper is organized as follows: the results of the electronic-structure calculations within the framework of the linear muffin-tin orbital method (LMTO $)^{21}$ at the LDA level are presented in section [II Comparison of $\mathrm{LDA}$ and $\mathrm{LDA}+\mathrm{U}$ results with experiments are also discussed in that section. In Sec. IA we describe the $a b$ initio construction of the many-body model Hamiltonian. Specifically, the uncorrelated part of the Hamiltonian for excitations in the vicinity of the Fermi level is obtained from the so-called downfolding technique ${ }^{22,23}$ within the Nth-order muffin tin orbital (NMTO) method. The interaction part is then estimated by the constrained-LDA method. In Sec. IIB we give a short summary of the VCA approach. We present and discuss our results in Sec. III. In particular, in Sec. IIIA, we evaluate the den- 
sity of states within VCA and compare it with experiments, and discuss the results in the framework of previous calculations. In Sec. IIIB we discuss $k$-dependent spectral properties, namely the spectral function and the self-energy, and evaluate the effective electron mass which we also compare to experiment. Finally, we summarize our results in Sec. IV

\section{ELECTRONIC STRUCTURE CALCULATIONS FOR TIN}

TiN crystallizes in the rock-salt (B1) structure where $\mathrm{Ti}$ and $\mathrm{N}$ atoms are sitting on interpenetrating facecentered cubic lattices originating at $(0,0,0)$, and at $\left(\frac{1}{2}, \frac{1}{2}, \frac{1}{2}\right)$ in units of the lattice parameter $a=7.65 a_{0}$ $\left(a_{0}=\right.$ Bohr radius), respectively. Each $\mathrm{Ti}(\mathrm{N})$ atom has six N (Ti) nearest neighbors in an octahedral geometry. Fig. 1 shows the conventional unit cell containing Ti (large spheres, red) and N (small sphere, blue) atoms. The electronic configuration of the outer shell of $\mathrm{Ti}$ is

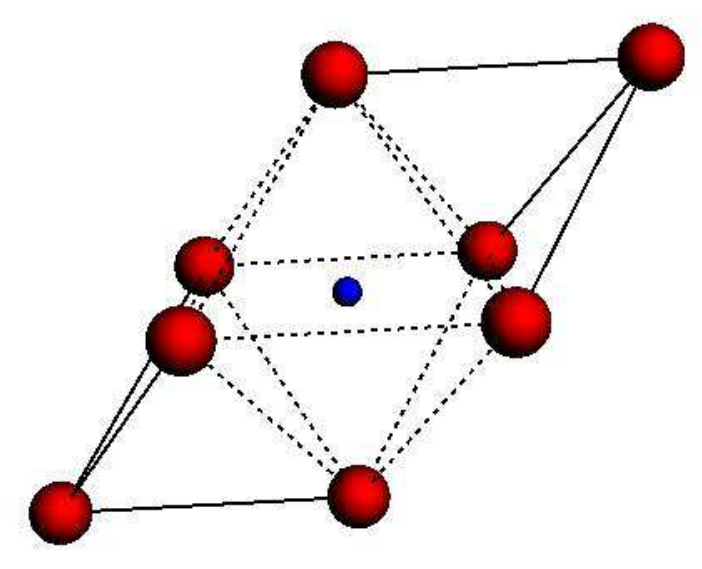

FIG. 1: (color online) Octahedral environment formed by the Ti-atoms (large, red spheres) around the $\mathrm{N}$ atom (small, blue sphere). The unit cell is represented by the black lines; the subset of Ti-atoms connected by dashed lines shows the cluster geometry that was used as reference system for the VCAcalculation (see text).

$4 s^{2} 3 d^{2}$. Therefore, following from the formal oxidation $\mathrm{Ti}^{3+}$, there is a single Ti electron in the valence band. Due to the octahedral symmetry, the Ti- $d$-orbitals are split into the three $t_{2 g}$ orbitals at lower energy, and two $e_{g}$ orbitals at higher energy.

The LDA band structure of TiN was computed with the LDA-LMTO (ASA) $\operatorname{code}^{21}$ which uses the basis of linearized muffin-tin orbitals in the atomic sphere approximation. Numerically, two empty spheres per unit cell have to be introduced at the positions $(1 / 4,1 / 4,1 / 4)$ and $(3 / 4,3 / 4,3 / 4)$, due to the atomic sphere approximation (ASA) used in the calculation. Results are shown in Fig. 2. In the density of states, the N-s orbital (not shown) form the lowest valence band widely separated from the other valence bands. At higher energies, one finds the set of bands formed by $\mathrm{N}-p$ orbitals situated between -10 and $-4 \mathrm{eV}$. Finally, the last five bands mainly consist of Ti-d orbitals. The DOS around the Fermi level is dominated by Ti- $t_{2 g}$ bands, while $e_{g}$ bands remain above the Fermi level and are empty. $t_{2 g}$ and $e_{g}$ bands overlap in the unoccupied part of the spectrum. On the other hand, the hybridization of Ti-d orbitals with N$p$ orbitals below the Fermi level is at the origin of the covalent bonding. Concerning the $p-d$ hybridization, the energetically higher $d$-bands form the anti-bonding states while the lower $p$-bands form the bonding states. The position of the Fermi level $\left(E_{F}\right)$ is determined by the number of valence electrons per unit cell being equal to 9 .

In order to take into account correlation effects on a mean field-level, we carried out an $\mathrm{LDA}+\mathrm{U}$ calculation. Here, we used values of $U=10 \mathrm{eV}$ and $J=1.3 \mathrm{eV}$, as obtained from the constrained LDA calculations ${ }^{24,25}$. As one can see there are no significant differences between the LDA and the $\mathrm{LDA}+\mathrm{U}$ results despite the large value of $U$. However, a comparison of these calculations with valence band XPS spectra, which provide a measure of the total density of occupied states as a function of energy, shows that neither LDA nor LDA $+\mathrm{U}$ results are appropriate to describe the experimental spectra in the vicinity of the Fermi energy unless one introduces ad-hoc broadening terms. Specifically, while the position of the $\mathrm{N}-p$ bands obtained by LDA and LDA $+\mathrm{U}$ is in reasonable agreement with the XPS measurements (see Fig 2), both methods fail to reproduce the behavior of the DOS within a range of $\approx 2 \mathrm{eV}$ below the Fermi energy. In particular, the XPS spectra show a local maximum at energies of $\approx-1 \mathrm{eV}$, followed by a "pseudogap"-like suppression at the Fermi energy. The $\mathrm{LDA}+\mathrm{U}$ results do not change qualitatively when decreasing $U$ down to $U \approx 8$, which, as argued below, is more appropriated for this material.

These results suggest that static inclusion of correlations is not sufficient to explain the DOS near the Fermi energy. For this reason, we have taken into account dynamical correlation effects beyond the LDA results by means of the VCA, a method appropriate to treat correlated systems. This approach builds up on the exact diagonalization of an Hamiltonian on a finite cluster combined with an appropriate extension to the infinite-lattice limit. However, in order to limit the size of the Hilbert space, it is necessary to use an effective Hamiltonian describing a small number of correlated effective orbitals per lattice site in the close vicinity of the Fermi level. From Fig. 2, we conclude that the minimal model has to be restricted to $\mathrm{Ti}\left(t_{2 g}\right)$ bands, while higher $\mathrm{Ti}\left(e_{g}\right)$ 


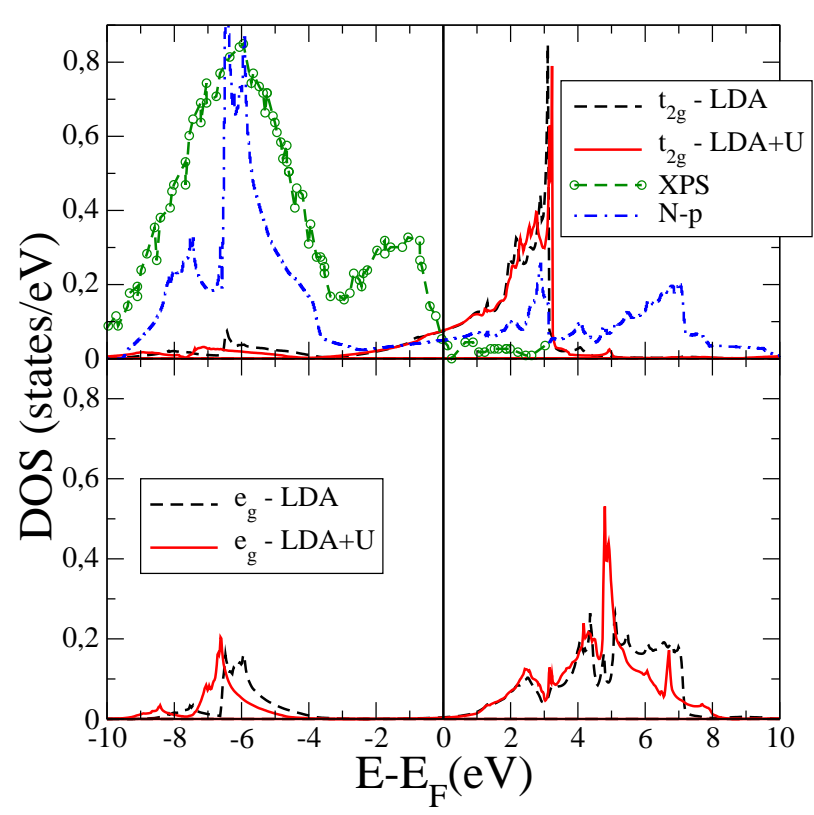

FIG. 2: (color online) Orbital-resolved density of states for $\mathrm{Ti}$ obtained by LDA and LDA+U. The upper (lower) panel shows the $t_{2 g}\left(e_{g}\right)$ contribution. The upper panel also displays the N- $p$ density of states and the experimental XPS spectrum ${ }^{3}$ (Experimental data reproduced with kind permission of the authors).

bands are unoccupied and can be neglected.

\section{A. Ab-initio construction of the model Hamiltonian}

In order to construct the effective low-energy Hamiltonian to use in our VCA calculation, we employed the Nth order muffin-tin-orbitals scheme within the same LMTOASA basis set. The NMTO method ${ }^{22,23}$ can be used to generate truly minimal basis sets with a massive downfolding technique. This reduced basis set reproduces the bands obtained with the full basis set to great accuracy and can thus be used as non-interacting part of the manybody Hamiltonian. According to the discussion in Sec. III the minimal basis set is obtained by downfolding to the $T i-d\left(t_{2 g}\right)$ manifold. The truly minimal set of symmetrically orthonormalized NMTOs is a set of Wannier functions. In the construction of this set, the active channels are forced to be maximally localized onto the eigenchannel $\mathbf{R} l m$ ( $\mathbf{R}=$ atomic position, $l, m=$ angular momentum quantum numbers), which makes the basis set strongly localized and suitable for a real-space Hamiltonian.

In this way, the non-interacting part of the effective Hamiltonian is confined to a reduced set of orbitals and to a reduced energy window. The NMTO downfolded bands are obtained by expanding around a small num- ber of energy points on which the LDA bands are reproduced exactly. To optimize the overall agreement with the energy bands, we chose the following expansion points $E_{\nu}-E_{F}=1.512 \mathrm{eV}, 0.016 \mathrm{eV}$, and $-0.664 \mathrm{eV}$. Results are quite stable for choices of the $E_{\nu}$ around these values. In Fig. 3 we show the eigenvalues of the effective Hamiltonian along some high-symmetry directions in comparison with the bands obtained from the full orbital basis. From this figure it is clear that the Ti-d $\left(t_{2 g}\right)$ manifold is well described by the non-interacting part of the effective Hamiltonian.

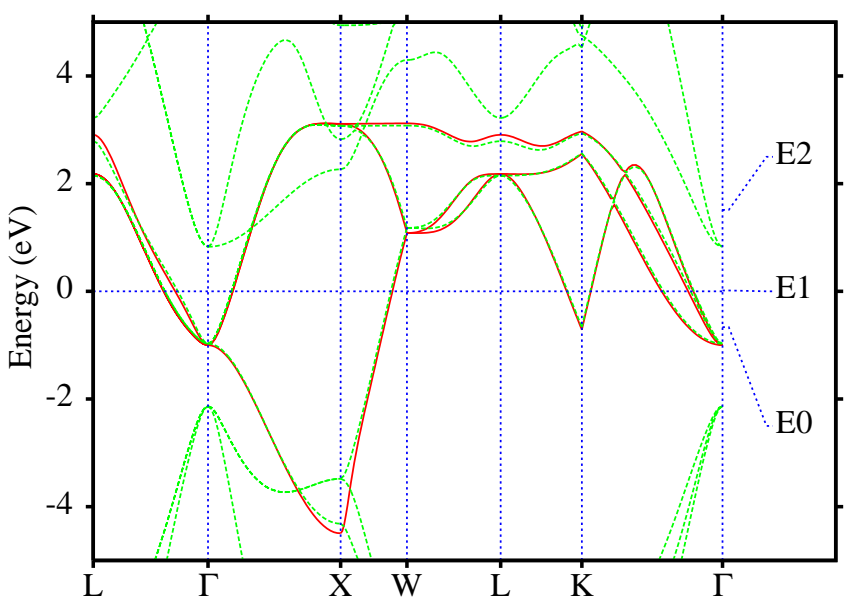

FIG. 3: (color online) Band-structure of TiN calculated for a path in the Brillouin zone (BZ) going from the L-point $(0.5,0.5,0.5)$ trough the $\Gamma(0,0,0), X(0,1,0), W(0.5,1,0)$, $L(0.5,0.5,0.5), K(0,0.75,0.75)$ points and ending at the $\Gamma$ point $(0,0,0)$. The bands are obtained from the LDA calculation (dashed/green line) for the complete orbital basis set. The NMTO bands (full/red line) are obtained after downfolding to the Ti-3d( $\left.t_{2 g}\right)$ orbitals.

Fourier-transformation of the orthonormalized NMTO Hamiltonian, $H^{\mathrm{LDA}}(\mathbf{k})$, yields on-site energies and hopping integrals $(\mathbf{R} \equiv(x, y, z))$,

$$
\left\langle\chi_{\mathbf{R}^{\prime} m^{\prime}}^{\perp}\left|H^{L D A}-\varepsilon_{F}\right| \chi_{\mathbf{R} m}^{\perp}\right\rangle \equiv t_{m^{\prime}, m}^{\mathbf{R}^{\prime}-\mathbf{R}}
$$

in a Wannier representation, where the NMTO Wannier functions $\left|\chi_{\mathbf{R} m}^{\perp}\right\rangle$ are orthonormal. In the restricted NMTO basis, $m$ is labeled by the three $t_{2 g}$ orbitals $m=x y, y z, z x$ (we use this order for the definition of the matrix elements below). In this basis, the on-site matrix elements $t_{m^{\prime}, m}^{000}$ are diagonal and independent of $m$. The precise value of these terms, as well as the socalled double-counting correction, are not important, as they can be absorbed into the chemical potential. The directional hopping matrix elements up to second nearest- 
neighbor are given by

$$
\begin{aligned}
t_{m^{\prime}, m}^{\frac{1}{2} 0} & =\left(\begin{array}{rrr}
-0.66 & 0 & 0 \\
0 & 0.15 & -0.1 \\
0 & -0.1 & 0.15
\end{array}\right), \\
t_{m^{\prime}, m}^{100} & =\left(\begin{array}{rrr}
-0.23 & 0 & 0 \\
0 & 0.01 & 0 \\
0 & 0 & -0.23
\end{array}\right),
\end{aligned}
$$

in units of eV. Only one representative hopping integral is shown for each class. Other hopping terms can be derived from proper unitary transformation using crystal symmetry. See, e.g., Ref. 26 for details.

Further hoppings are taken into account up to a range of $r=1.1 a$. Neglected hoppings are at least by a factor 40 smaller than the largest nearest-neighbor hopping.

In order to make sure that neglecting $e_{g}$ orbitals is safe, we have also carried out a LDA+VCA calculation (for smaller clusters) using an Hamiltonian downfolded to all $5 \mathrm{Ti}$-d bands. We have verified that the occupation of the $e_{g}$ bands is less than $10^{-3}$ per Ti-atom, so that the corresponding Wannier functions can be safely neglected in our calculation. Notice, however, that, due to hybridization, $t_{2 g}$ Wannier functions also have a certain amount of $e_{g}$ character.

The non-interacting part of the effective Hamiltonian for TiN, thus, has the form

$$
H_{0}=\sum_{\mathbf{R}^{\prime}, \mathbf{R},\left\{m^{\prime}, m\right\}, \sigma} t_{m^{\prime}, m}^{\mathbf{R}^{\prime}-\mathbf{R}} c_{\mathbf{R}^{\prime} m^{\prime} \sigma}^{\dagger} c_{\mathbf{R} m \sigma} .
$$

To take into account correlation effects, we add the usual interaction term

$$
\begin{aligned}
H_{I} & =\sum_{\mathbf{R}, m} U n_{\mathbf{R} m \uparrow} n_{\mathbf{R} m \downarrow} \\
& +\sum_{\mathbf{R}, m<m^{\prime}, \sigma, \sigma^{\prime}}\left(U^{\prime}-J \delta_{\sigma, \sigma^{\prime}}\right) n_{\mathbf{R} m \sigma} n_{\mathbf{R} m^{\prime} \sigma^{\prime}} \\
& +\sum_{\mathbf{R}, m<m^{\prime}} J c_{\mathbf{R} m^{\prime} \uparrow}^{\dagger} c_{\mathbf{R} m \downarrow}^{\dagger} c_{\mathbf{R} m^{\prime} \downarrow} c_{\mathbf{R} m \uparrow}+\text { h.c. } \\
& +\sum_{\mathbf{R}, m<m^{\prime}} J c_{\mathbf{R} m^{\prime} \uparrow}^{\dagger} c_{\mathbf{R} m^{\prime} \downarrow}^{\dagger} c_{\mathbf{R} m \downarrow} c_{\mathbf{R} m \uparrow}+\text { h.c.. }
\end{aligned}
$$

In Eq. 3. $c_{\mathbf{R} m \sigma}\left(c_{\mathbf{R} m \sigma}^{\dagger}\right)$ are the usual fermionic annihilation (creation) operators acting on an electron with spin $\sigma$ at site $\mathbf{R}$ in the orbital $m$ and $n_{\mathbf{R} m \sigma}=c_{\mathbf{R} m \sigma}^{\dagger} c_{\mathbf{R} m \sigma}$. $U$ denotes the Coulomb-interaction for two electrons in the same orbital with anti-parallel spin. If they are located on two different orbitals, the interaction is reduced to $U^{\prime}=U-2 J . J$ is the Hund's rule exchange constant and h.c. denotes the hermitian conjugate.

We have estimated the value of the Coulombinteraction parameter by means of constrained LDA, $, 24,25$ whereby occupancies on all $d$ orbitals have been fixed. Since the $e_{g}$ effective orbitals are essentially empty, as discussed above, they do not contribute to screening and can be safely neglected. The constrained calculation yields a value of $U \approx 10 \mathrm{eV}$ and $J \approx 1.3 \mathrm{eV}$. However, as it was shown by Aryasetian et al ${ }^{27}$, constrained LDA gives larger values for $U$ compared to other methods based on the evaluation of the screened Coulomb interaction within the Random Phase Approximation ${ }^{28}$. Therefore, we have also investigated smaller $U$ values. We will show below that results change drastically around $U \approx 9 \mathrm{eV}$, where a Mott-insulator transition takes place.

As correlations on a mean-field level are already included in LDA, one should in principle subtract the long discussed double-counting 29,30 correction. However, since $t_{2 g}$ orbitals are degenerate, this correction is simply a constant that can be absorbed in the chemical potential.

\section{B. Variational Cluster Approach}

To solve the many-body Hamiltonian (2) $+(3)$ we employ the Variational Cluster Approach19,20. This method is an extension of Cluster Perturbation Theory $(\mathrm{CPT})^{31,32,33}$, in which the original lattice is divided into a set of disconnected clusters and the inter-cluster hopping terms are treated perturbatively. VCA additionally includes "virtual" single-particle terms to the cluster Hamiltonian, yielding a so-called reference system, and then subtracts these terms perturbatively. The "optimal" value for these variational parameters is determined in the framework of the Self-energy Functional Approach (SFA) ${ }^{34,35}$, by requiring that the SFA grand-canonical potential $\Omega$ is stationary within this set of variational parameters. Since TiN is paramagnetic, we only include the chemical potential of the cluster as a variational parameter. The latter is necessary in order to obtain a thermodynamically consistent particle density 36,37 . In this paper, we use a new method, described in Ref. 38, to carry out the sum over Matsubara frequencies required in the evaluation of $\Omega$, whereby an integral over a contour lying a distance $\Delta$ from the real axis is carried out. The crucial point is that the contour integral is exact for any (even large) $\Delta$.

As a reference system we adopt the minimal cluster that contains the full lattice symmetry. This consists of all $6 \mathrm{Ti}$ sites lying on the corners of an octahedron, as shown in Fig. 1. Larger clusters are at present not feasible within our variational procedure. As in the case of cluster-DMFT (which is currently not feasible for a 6 -site cluster with three orbitals each), the appropriate periodisation is a crucial issue ${ }^{39,40}$. Here, we choose to periodise the Green's function, as it is well known that the self-energy periodisation gives unphysical results in the vicinity of an insulating phase. 


\section{RESULTS}

\section{A. Density of states}

As discussed in Sec. III, the low-energy XPS ${ }^{3}$ spectrum is characterized by a peak at about $-1 \mathrm{eV}$ followed by a "pseudogap", i. e. a suppression of states at the Fermi level. This latter fact is consistent with the K-ELNES spectrum ${ }^{41}$, which provides information about the DOS above the Fermi energy (cf. Fig. 5). This suppression is not reproduced by LDA electronic structure calculations, suggesting that strong electronic correlations may play an important role for this material. In a previous work ${ }^{3}$, it was suggested, in order to improve the agreement with the measured spectra, to convolute the computed DOS with a combination of a Lorentzian and a Gaussian curve, taking into account the broadening due to lifetime and experimental resolution effects. This treatment indeed improves on the overall shape producing a peak at an energy of about $-1.5 \mathrm{eV}$, although one should point out that the fitted broadening parameters are much too large (around 0.6 and $0.8 \mathrm{eV}$, respectively).

In the present paper, we argue that the pseudogap observed in the DOS of stoichiometric TiN is intrinsic to this material and signals the proximity to a Mott metalinsulator transition. To show this we start by calculating the DOS obtained from the Hamiltonian (3) with the value of $U=10 \mathrm{eV}$ obtained from constrained LDA. The results, displayed in Fig. 4, predict for this value of $U$ a Mott-insulating state with a gap of about $1 \mathrm{eV}$. However, this is in contrast to the experimental situation, since electrical conductivity in TiN shows a metallic behavior, although with a relatively low residual conductivity ${ }^{42}$. Since results obtained from constrained LDA are only approximate and tend to overestimate $U$ due to the delocalized nature of Wannier orbitals, we have also presented results for smaller values of $U$ down to $8 \mathrm{eV}$ (Fig. 4). As one can see, no significant changes can be detected for states more than $3 \mathrm{eV}$ away from the Fermi energy. Here, only static correlations, which get absorbed into the chemical potential, play a role. On the other hand, the situation changes rapidly around the Fermi energy. In particular, our results show that the Mott gap starts closing at $U \approx 9 \mathrm{eV}$, and at smaller $U$ down to $U \approx 8 \mathrm{eV}$ it acquires the shape of a pseudogap. As a matter of facts, the curve for $U=8.5$, shown in Fig. [5] agrees quite well with experimental measurements. Notice that, in order to avoid introducing $a d-h o c$ parameters, and to show fine-detailed features of the spectrum, the calculated curve has not been additionally broadened to meet experimental resolution. Taking this into account, we see that our results reproduce quite well the experimental features both above (K-ELNES) and below (XPS) the Fermi energy, and in particular the "pseudogap" behavior between -1.0 and $1.0 \mathrm{eV}$. We stress that we are using an effective low-energy model which is expected to correctly reproduce correlation effects close to the Fermi energy, but is not expected to reproduce features beyond the range of the NMTO bands shown in Fig. 3 (full/red curves). To reproduce the spectrum at higher binding energies, $\mathrm{LDA}$ and $\mathrm{LDA}+\mathrm{U}$ are more appropriate. In this sense, our results complement these techniques in the low-energy region.

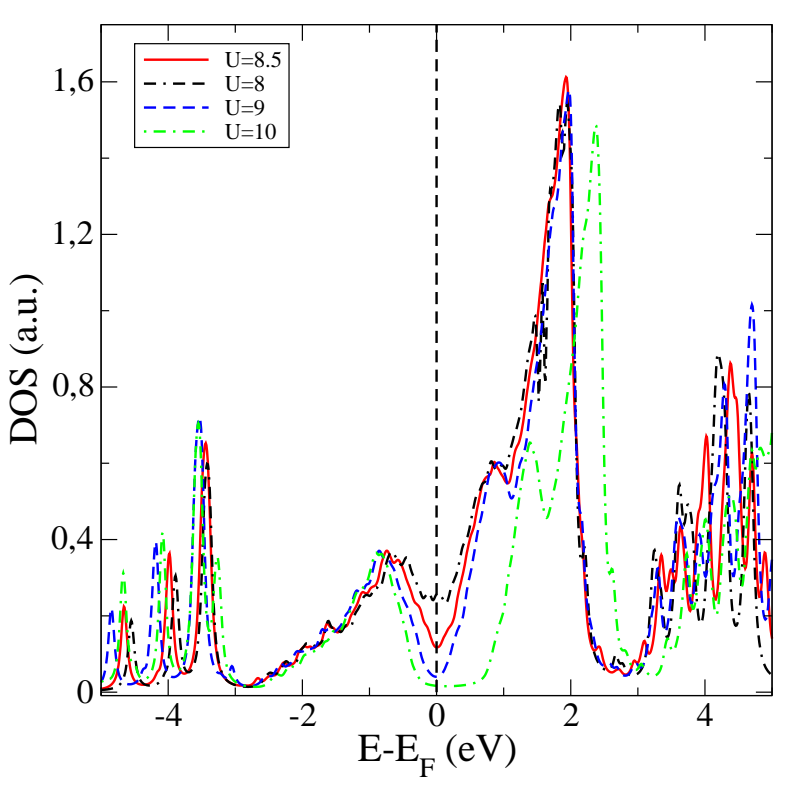

FIG. 4: (color online) TiN density of states calculated with LDA+VCA for different values of the Coulomb-interaction parameter $U$ and for $J=1.3$.

Another remarkable feature of the computed $\mathrm{LDA}+\mathrm{VCA}$ density of states spectra is the presence of a set of peaks in the energy range of -5 to $-3 \mathrm{eV}$. These states only appear when a sufficiently large cluster is taken as a reference system. Therefore, these states can be seen as non-local many-body incoherent features. It is tempting to associate these states with the corresponding "kink" in the XPS spectrum 3 at energies around $-4 \mathrm{eV}$. However, this kink is also affected by the presence of $\mathrm{N}-2 p$ states whose bands start at $\approx-4 \mathrm{eV}$ (see Fig. 2). Therefore, our result suggests a strong hybridization between these states and the correlated many-body structures at $\approx-4 \mathrm{eV}$.

The nitrogen K-ELNES spectrum is related to the unoccupied partial density of states with $p$-symmetry at the nitrogen site. The peaks situated at energies around 2 and $4 \mathrm{eV}$ can be attributed to the unoccupied N-2p states hybridized with Ti $3 d$ states. Notice that the downfolded operators used in our effective Hamiltonian (2) + (3) describe anyway an effective particle ultimately producing the bands of Fig. 3, i.e. the corresponding particle also contain a "mixture" of other orbitals such as N- $p$, in order to correctly reproduce the hybridization. It is remarkable to note that our calculation with $U=8.5 \mathrm{eV}$ captures most of the features of the experimental spectra at both peaks, in particular the separation of the two peaks is in very good agreement with the experimental 


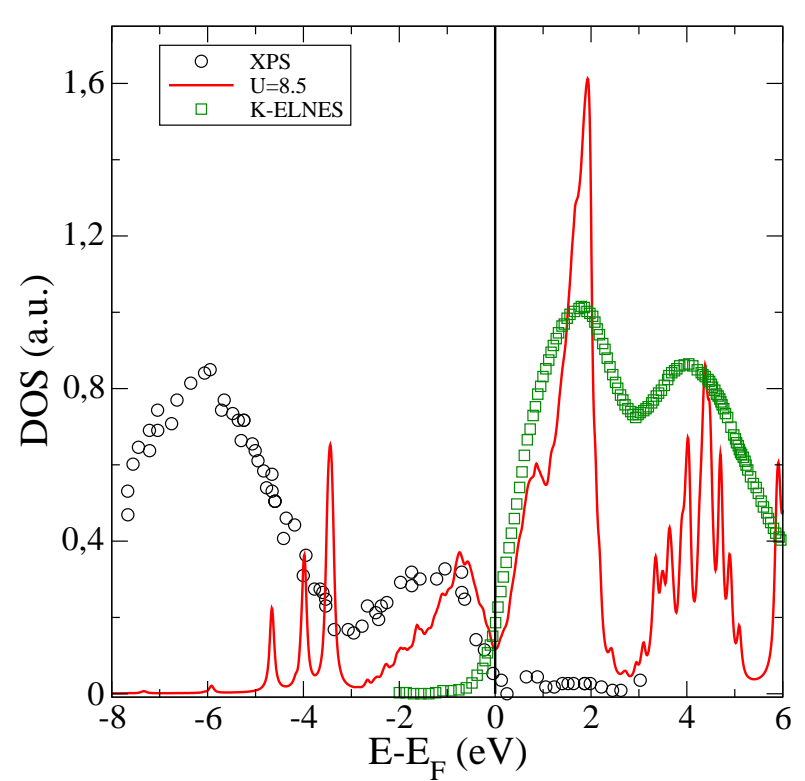

FIG. 5: (color online) TiN density of states calculated within $\mathrm{LDA}+\mathrm{VCA}$ for $U=8.5 \mathrm{eV}$ and $J=1.3$ compared with measured $\mathrm{XPS}^{3}$ and K-ELNES ${ }^{17}$ spectra (Experimental data reproduced with kind permission from the authors).

value $2.3 \mathrm{eV} \underline{17}$.

It is important to mention that combining the experimental data from the occupied and unoccupied parts of the spectra with electric and magnetic properties of $\mathrm{TiN}^{42}$, a peculiar metallic behavior emerges. Magnetic susceptibility measurements show that TiN is a Pauliparamagnet, and electrical conductivity demonstrates a metallic behavior with a relatively large residual resistivity. In combination with the measured XPS spectra, one can conclude that at the Fermi level a pseudogap in the density of states is formed, signaling the vicinity of a Mott-insulating phase.

The present LDA+VCA calculation includes correlation effects exactly on a length scale of an octahedral cluster consisting of 6 sites shown in Fig. 1 (connected by dashed lines) . Notable results of our present calculations are the correct description of the $-1 \mathrm{eV}$ peak and the double peak in the occupied part of the spectra. In addition, at lower energies, many-body non-local incoherent features are formed which can explain the kink in the XPS spectra situated at $-4 \mathrm{eV}$. Therefore, it is clear that the present LDA+VCA results show notable improvements with respect to previous DFT-results in the low-energy region, and explain the peculiar metallic behavior of TiN.

A remark should be made concerning vacancy effects. It is well known that transition metal carbides and nitrides usually contain vacancies in the metalloid (N) sublattice. The presence of vacancies profoundly influences the physical properties of this family of compounds. It is known from electronic-structure calculations that the presence of vacancies reduces the partial nitrogen $s$ and $p$ density of states, and produces additional peaks close to the Fermi level. These vacancy peaks show up in the LDA-DOS of non-stoichiometric TiN at about $-2 \mathrm{eV}$ and in the vicinity of the Fermi level $15,16,43,44$. The peak at $-2 \mathrm{eV}$ is associated with $\sigma$-bonding between Ti atoms through the $\mathrm{N}$ vacancy, while the peak at $E_{F}$ is related to the $\sigma$-bonding between the nearest-neighbor $\mathrm{Ti}$ atoms 15.43 . A quantitative comparison of the measured spectra and the broadened density of states obtained for non-stoichiometric materials shows that the calculated density of states peaks are too narrow and shifted to lower binding energies with respect to experimental results. The shift and the narrowing of the theoretical peaks was interpreted $\frac{45}{5}$ as due to limitations of the local density approximation in describing electronic interactions.

\section{B. Spectral properties}

In order to gain insight into $\mathbf{k}$-dependent features of the local DOS, we have computed the $\mathbf{k}$-resolved spectral function $A(\mathbf{k}, \omega)$, which is plotted in Fig. 6. From this figure one can clearly discern two metallic bands crossing the Fermi energy near to the $\mathrm{W}$ point, and one between $K$ and $\Gamma$, however, with a small spectral weight, consistent with the pseudogap picture discussed in Sec. IIIA.

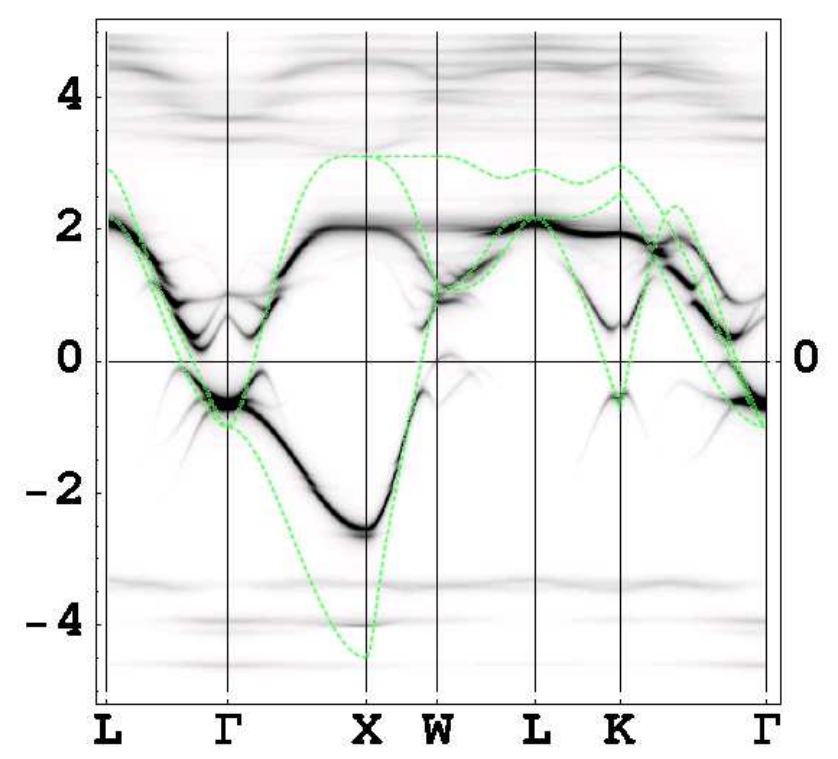

FIG. 6: Spectral-function of TiN for $U=8.5$ and $J=1.3$ shown as density plot for the same path in the BZ as in Fig. 3. The bands obtained from downfolding (dashed, green lines) are shown as reference.

Moreover, in the energy range between -3 to $-5 \mathrm{eV}$ the spectral function in Fig. 6] shows dispersion-less features which are responsible for the non-local correlation peaks discussed in the previous section. As discussed above, these features cannot be captured within a single- 
site LDA+Dynamical-Mean-Field (DMFT) approach, so that a cluster approach, as the one presented here, is required. Also the cluster geometry itself is important: we chose an octahedral reference cluster (Fig. (1) in order to conserve the lattice symmetry. This is needed, as we have verified that in smaller clusters, without the lattice symmetry, this feature is not present.

As discussed above, our calculations show that dynamical correlations are important for TiN. On the other hand, it turns out that also non-local correlations are crucial. An important consequence of this fact is that we do not expect the properties of TiN discussed here to be correctly reproduced by a single-site DMFT calculation.

To show this, we plot in Fig. 7 the self-energy on the same path around the BZ as for the spectral function (Fig. 6). As one can see, there is a strong $\mathbf{k}$ dependence, especially in the region around the Fermi energy. This is an indication for the non-locality of the self-energy. This non-locality strongly affects the metal-insulator transition as well, since the most dispersive part of $\Sigma$ is precisely around the Fermi energy. Furthermore, there are contributions to the self-energy at larger energies (not shown in Fig. 7), which, however, are essentially flat as a function of $\mathbf{k}$, and, thus, localized in real space.

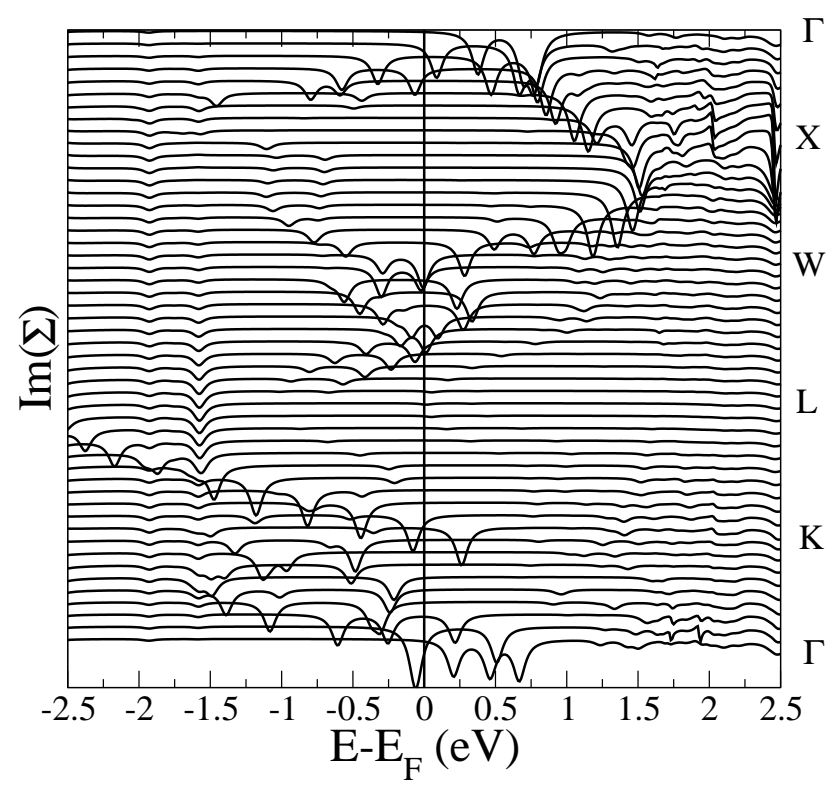

FIG. 7: Imaginary part of the self-energy calculated for the same parameters and along the same BZ path as in Fig. 6] and presented in a three-dimensional plot.

The k-resolved spectral function allows us to determine the LDA+VCA quasiparticle bands, which are shown as density plot in Fig. 6 and are compared with the bands obtained from LDA (dashed). One can observe a reduction of the quasiparticle bandwidth (BW) from approximately $7.5 \mathrm{eV}$ in LDA to $4.5 \mathrm{eV}$ in $\mathrm{LDA}+\mathrm{VCA}$. Accordingly, the band narrowing due to correlation effects can be described in terms of a "high-energy" (HE) massrenormalization factor $m_{H E} / m_{H E, L D A} \approx 1.7$. Experimental measurements provide the total ratio $m_{H E} / m_{e}$ ( $m_{e}$ is the free electron mass), which contains also the mass renormalization factor $m_{H E, L D A} / m_{e}$ coming from the band structure. The latter can be roughly estimated by equating $\hbar^{2} k_{F}^{2} /\left(2 m_{H E, L D A}\right)=E_{F}^{*}$, whereby $E_{F}^{*}=4.42 \mathrm{eV}$ is the Ti-3d band "depth", i. e. the Fermi energy measured from the bottom of the occupied Ti-3d band, and $k_{F}$ is related to the corresponding occupation $n=4 / a^{3}$ by the usual relation $k_{F}^{3}=3 \pi^{2} n$. This gives $m_{H E, L D A} / m_{e} \approx 1.3$, producing an overall high-energy mass renormalization $m_{H E} / m_{e}=2.2$. This has to be distinguished from the low-energy effective mass given by the change in slope at the Fermi energy, i. e. the Fermi velocity. Nevertheless, available experimental measurements discussed below provide an estimate for $m_{H E}$ and not for the low-energy contribution.

Experimentally, an estimate of the electron effective mass in TiN has been obtained by UPS and EELS experiments using high-energy synchrotron radiation ${ }^{46}$. These methods also allow to determine the band density $n$ of the $d$-electrons and the $3 d$ band depth (corresponding to our $E_{F}^{*}$ above). To estimate the $d$-electron density several characteristics of the UPS spectrum such as peak area, photoemission cross section and inelastic mean free path are used. The estimation of the band depth is obtained from the position of the minimum in intensity between the valence and conduction bands ${ }^{46}$. Considering the rough estimate we have used, the computed high-energy mass renormalization factor $m_{H E} / m_{e}=2.2$, turns out to be in reasonable agreement with the value $2.7 \pm 0.3$, obtained in Ref. 46, table 3, for the stoichiometric TiN sample d.

\section{SUMMARY}

In this paper we have analyzed the physical properties of TiN in a combined electronic-structure and many-body approach. The NMTO downfolding technique was used to calculate the LDA low-energy effective Hamiltonian for the $T i-d\left(t_{2 g}\right)$ bands. The effect of non-local correlations was treated within a variational cluster perturbation approach, which deals exactly with correlations on the cluster scale. We present results for the local density of states, the self-energy, the spectral function and for the effective mass. The results are analyzed in comparison with experiments on valence band XPS and K-ELNES spectra and, in the case of the effective mass, compared to UPS and EELS experiments.

Our results suggest that TiN is a peculiar metal with a pseudogap at the Fermi level indicating the proximity to a metal-insulator transition. In our calculations the pseudogap regime is best described for a value of $U=$ $8.5 \mathrm{eV}$ for the Coulomb-interaction.

It is important to mention that this result could be achieved only by an appropriate treatment of dynam- 
ical and non-local correlations. In particular, neither LDA nor LDA+U calculations provide even a qualitative description of the DOS suppression at the Fermi energy. Due to the non-locality of the self-energy, we expect that even single-site DMFT may not be appropriate for a proper description of this system at low energies.

Our calculations provide a good qualitative and semiquantitative agreement with experiments, when comparing the DOS with XPS and K-ELNES spectra close to the Fermi energy. In particular, our results show a coherent feature situated at $-1 \mathrm{eV}$ which can be clearly identified in the XPS spectra. We stress that none of previous theoretical investigations are able to capture this particular low energy feature. Furthermore, at low energies our results provide a qualitative interpretation of the $-4 \mathrm{eV}$ kink in the XPS spectra as an hybridization of incoherent non-local many-body features with the N-p states. Also, in the unoccupied part above the Fermi level, our results reproduce the double peak structure in good agreement with the K-ELNES spectra. Finally, we calculated the effective mass from high- and low-energy spectral features and found reasonable agreement with experimental values.

\section{Acknowledgments}

We are grateful to Karsten Held for helpful suggestions. This work was supported by the Austrian science fund (FWF project P18505-N16), and by the cooperation project "NAWI Graz" (F-NW-515-GASS). L.C. also acknowledges the financial support offered by Romanian Grant CNCSIS/ID672/2009.
* hannes.allmaier@itp.tugraz.at

1 K. Schwarz, A. R. Williams, J. J. Cuomo, J. H. E. Harper, and H. T. G. Hentzell, Phys. Rev. B 32, 8312 (1985).

2 V. M. Vinokur, T. I. Baturina, M. V. Fistul, A. Y. Mironov, M. R. Baklanov, and C. Strunk, Nature 452, 613 (2008).

3 L. Soriano, M. Abbate, H. Pen, P. Prieto, and J. M. Sanz, Solid State Communications 102, 291 (1997).

4 L. Porte, L. Roux, and J. Hanus, Phys. Rev. B 28, 3214 (1983).

5 L. I. Johansson, P. M. Stefan, M. L. Shek, and A. N. Christensen, Phys. Rev. B 22, 1032 (1980).

6 V. A. Gubanov, E. Z. Kurmaev, and G. P. Shveikin, J. Phys. Chem. Solids 38, 201 (1977).

7 F. Riehle, T. Wolf, and C. Politis, Z. Phys. B 47, 3 (1982).

${ }^{8}$ W. K. Schubert, R. N. Shelton, and E. L. Wolf, Phys. Rev. B 23, 5097 (1981).

9 W. K. Schubert, R. N. Shelton, and E. L. Wolf, Phys. Rev. B 24, 6278 (1981).

10 D. W. Lynch, C. G. Olson, D. J. Peterman, and J. H. Weaver, Phys. Rev. B 22, 3991 (1980).

11 J. F. Alward, C. Y. Fong, M. El-Batanouny, and F. Wooten, Phys. Rev. B 12, 1105 (1975).

12 A. Neckel, International Journal of Quantum Chemistry 13, 1317 (1983).

13 P. Marksteiner, P. Weinberger, A. Neckel, R. Zeller, and P. H. Dederichs, Phys. Rev. B 33, 812 (1986).

14 D. A. Papaconstantopoulos, W. E. Pickett, B. M. Klein, and L. L. Boyer, Phys. Rev. B 31, 752 (1985).

15 V. P. Zhukov, V. A. Gubanov, O. Jepsen, N. E. Christensen, and O. K. Andersen, J. Phys. Chem. Solids 49, 841 (1988).

16 S.-H. Jhi, S. G. Louie, M. L. Cohen, and J. Ihm, Phys. Rev. Lett. 86, 3348 (2001).

17 A. T. Paxton, M. van Schilfgaarde, M. MacKenzie, and A. J. Craven, J. Phys.: Condens. Matter 12, 729 (2000).

18 V. I. Anisimov, F. Aryasetiawan, and A. I. Lichtenstein, Journal of Physics: Condensed Matter 9, 767 (1997).

19 M. Potthoff, M. Aichhorn, and C. Dahnken, Phys. Rev. Lett. 91, 206402 (2003).

20 C. Dahnken, M. Aichhorn, W. Hanke, E. Arrigoni, and
M. Potthoff, Phys. Rev. B 70, 245110 (2004).

21 O. K. Andersen and O. Jepsen, Phys. Rev. Lett. 53, 2571 (1984).

22 O. K. Andersen and T. Saha-Dasgupta, Phys. Rev. B 62, R16219 (2000).

23 E. Zurek, O. Jepsen, and O. K. Andersen, ChemPhysChem 6, 1934 (2005).

24 V. I. Anisimov and O. Gunnarsson, Phys. Rev. B 43, 7570 (1991).

25 V. I. Anisimov, J. Zaanen, and O. K. Andersen, Phys. Rev. B 44, 943 (1991).

26 E. Pavarini, A. Yamasaki, J. Nuss, and O. K. Andersen, New J. Phys. 7, 188 (2005).

27 F. Aryasetiawan, K. Karlsson, O. Jepsen, and U. Schönberger, Phys. Rev. B 74, 125106 (2006).

${ }^{28}$ F. Aryasetiawan, M. Imada, A. Georges, G. Kotliar, S. Biermann, and A. I. Lichtenstein, Phys. Rev. B 70, 195104 (2004).

29 M. T. Czyzyk and G. A. Sawatzky, Phys. Rev. B 49, 14211 (1994).

30 A. G. Petukhov, I. I. Mazin, L. Chioncel, and A. I. Lichtenstein, Phys. Rev. B 67, 153106 (2003).

31 C. Gros and R. Valenti, Phys. Rev. B 48, 418 (1993).

32 D. Sénéchal, D. Perez, and M. Pioro-Ladriere, Phys. Rev. Lett. 84, 522 (2000).

33 S. G. Ovchinnikov and I. S. Sandalov, Physica C 161, 607 (1989).

34 M. Potthoff, Eur. Phys. J. B 32, 429 (2003).

35 M. Potthoff, Eur. Phys. J. B 36, 335 (2003).

36 M. Aichhorn and E. Arrigoni, Europhys. Lett. 72, 117 (2005).

37 M. Aichhorn, E. Arrigoni, M. Potthoff, and W. Hanke, Phys. Rev. B 74, 024508 (2006).

38 X. Lu and E. Arrigoni (2009), arXiv:0902.0388.

39 G. Biroli, O. Parcollet, and G. Kotliar, Phys. Rev. B 69, 205108 (2004).

40 D. Sénéchal (2008), arXiv:0806.2690.

41 M. Tsujimoto, H. Kurata, T. Nemoto, S. Isoda, S. Terada, and K. Kaji, Journal of Electron Spectroscopy and Related Phenomena 143, 161 (2005).

42 P. S. Herle, M. S. Hegde, N. Y. Vasathacharyan, and 
S. Philip, J. Solid. Stat. Chem. 134, 120 (1997).

43 P. Herzig, J. Redinger, R. Eibler, and A. Neckel, J. Solid. Stat. Chem. 70, 281 (1987).

44 Z. Dridi, B. Bouhafs, P. Ruterana, and H. Aourag, J. Phys.: Condens. Matter 14, 10237 (2002).
45 J. Redinger, P. Marksteiner, and P. Weinberger, Z. Phys. B 63, 321 (1986).

46 C. G. H. Walker, J. A. D. Matthew, C. A. Anderson, and N. M. D. Brown, Surf. Sci. 412/413, 405 (1998). 